\title{
State-Feedback Control of LPV Sampled-Data Systems
}

\author{
K. TAN and K.M. GRIGORIADIS* \\ Department of Mechanical Engineering, University of Houston, \\ Houston, TX77204, USA
}

(Received 14 December 1998)

In this paper, we address the analysis and the state-feedback synthesis problems for linear parameter-varying (LPV) sampled-data control systems. We assume that the statespace data of the plant and the sampling interval depend on parameters that are measurable in real-time and vary in a compact set with bounded variation rates. We explore criteria such as the stability, the energy-to-energy gain (induced $L^{2}$ norm) and the energy-to-peak gain (induced $L^{2}$-to- $L^{\infty}$ norm) of such sampled-data LPV systems using parameter-dependent Lyapunov functions. Based on these analysis results, the sampleddata state-feedback control synthesis problems are examined. Both analysis and synthesis conditions are formulated in terms of linear matrix inequalities that can be solved via efficient interior-point algorithms.

Keywords: Parameter-varying systems; Sampled-data systems;

Linear matrix inequalities

\section{INTRODUCTION}

The control of engineering systems often involves a continuous-time plant controlled using discrete-time measurements via analog-to-digital (A/D) and digital-to-analog (D/A) devices for interfacing. Sampleddata control has recently received increased attention to address the analysis and feedback control synthesis for these systems with guarantees for stability, performance and intersample behavior $[3,5,6,8]$. To this

\footnotetext{
* Corresponding author. Tel.: 713-743-4387. Fax: 713-743-4503.

E-mail: karolos@uh.edu.
} 
end, $H^{2}, H^{\infty}$ and $l^{1}$ control methodologies have been developed in a sampled-data framework using lifting techniques to associate a timeinvariant discrete-time system with infinite-dimensional input/output spaces to the initial sampled-data one, such that system induced norms are preserved.

In this work, the analysis and state-feedback control synthesis problems for linear parameter-varying (LPV) sampled-data systems are examined. The LPV systems theory $[1,2,10,11,16]$ has been motivated by the gain-scheduling approach for control of linear and nonlinear systems [12]. It provides a systematic methodology to design parameterdependent controllers that guarantee stability and performance specifications using computationally efficient linear matrix inequality (LMI) optimization tools. Hence, in our work, we assume that the state-space data of the sampled-data system and the length of the sampling interval depend on system parameters that are available for measurement in real time at the sampling instances. The dependency of the sampling interval on measurable system parameters allows the treatment of systems with variable sampling rates, such as engines, manufacturing systems and telerobotic systems. For example, in an internal combustion engine, the sampling interval is variable and depends on the engine speed (event-based sampling).

We seek to develop controllers that are scheduled based on the measurement of the parameters to guarantee stability and desired performance specifications. Two performance objectives are examined in this work: the energy-to-energy gain (induced $L^{2}$ norm) and the energy-topeak gain (induced $L^{2}$-to- $L^{\infty}$ norm). A lifting approach $[3,5]$ is followed to transform the sampled-data system to a discrete-time system with infinite-dimensional input/output spaces. Due to the parameter dependence of the system matrices and the sampling interval, the lifted system is a discrete-time LPV system with infinite dimensional input and output spaces. Parameter-dependent Lyapunov functions are utilized to develop analysis and state-feedback control synthesis results. The analysis and synthesis conditions are formulated in terms of parameter-dependent LMIs that can be discretized over the parameter space and solved using efficient interior point optimization algorithms. For the special case where the parameter set is a singleton, our results provide analysis and synthesis conditions for standard (parameter-independent) sampled-data systems in terms of LMIs. 
The notation to be used in this paper is as follows: The $L^{2}$ norm of a vector valued function $f(t)$ is $\|f\|_{2}=\left\{\int_{0}^{\infty} f^{\prime}(t) f(t) \mathrm{d} t\right\}^{1 / 2}$ and the $L^{\infty}$ norm is $\|f\|_{\infty}=\sup _{t}\left\{f^{\prime}(t) f(t)\right\}^{1 / 2}$. For a discrete-time vector valued signal $f(k)$, the $l^{2}$ norm is $\|f\|_{2}=\left\{\sum_{0}^{\infty} f^{\prime}(k) f(k)\right\}^{1 / 2}$ and the $l^{\infty}$ norm is $\|f\|_{\infty}=\sup _{k}\left\{f^{\prime}(k) f(k)\right\}^{1 / 2} \cdot(\cdot)^{\prime}$ stands for the transpose of a real matrix and $(\cdot)^{*}$ denotes the adjoint of a operator on Hilbert space. The matrix norm $\|\cdot\|$ is the maximum singular value of a matrix, that is $\|A\|=\bar{\sigma}(A)=\left\{\lambda_{\max }\left(A A^{\mathrm{T}}\right)\right\}^{1 / 2}$. Given a real matrix $B$, the orthogonal complement $B^{\perp}$ is defined as the (possibly non-unique) matrix that satisfies $B^{\perp} B=0$ and $B^{\perp} B^{\perp \mathrm{T}}>0$. Hence, $B^{\perp}$ can be computed from the singular value decomposition of $B$ as follows: $B^{\perp}=T U_{2}^{\mathrm{T}}$ where $T$ is an arbitrary nonsingular matrix and $U_{2}$ is defined from the singular value decomposition

$$
B=\left[\begin{array}{ll}
U_{1} & U_{2}
\end{array}\right]\left[\begin{array}{cc}
\Sigma_{1} & 0 \\
0 & 0
\end{array}\right]\left[\begin{array}{c}
V_{1}^{\mathrm{T}} \\
V_{2}^{\mathrm{T}}
\end{array}\right] .
$$

The standard notation $>, \geq(<, \leq)$ is used to denote the positive (negative) definite and semidefinite ordering of matrices.

\section{PRELIMINARIES}

Consider the following continuous-time LPV plant

$$
\begin{aligned}
\dot{x}(t) & =A(p(t)) x(t)+B_{1}(p(t)) w(t)+B_{2}(p(t)) u(t) \\
z(t) & =C_{1}(p(t)) x(t)+D_{11}(p(t)) w(t)+D_{12}(p(t)) u(t) \\
y(t) & =x(t)
\end{aligned}
$$

where $x(t) \in \mathbb{R}^{n}$ is the state vector, $w(t) \in \mathbb{R}^{m_{1}}$ is the vector of exogenous inputs, $u(t) \in \mathbb{R}^{m_{2}}$ is the vector of control inputs, $z(t) \in \mathbb{R}^{p_{1}}$ is the vector of controlled outputs, and $y(t) \in \mathbb{R}^{n}$ is the vector of measured outputs. We assume that the state-space matrices $A(\cdot), B_{1}(\cdot), B_{2}(\cdot), C_{1}(\cdot), D_{11}(\cdot)$ and $D_{12}(\cdot)$ are bounded continuous functions of a time-varying parameter vector $p(t) \in F_{\mathcal{R}}^{v}$. The set $F_{\mathcal{R}}^{v}$ is the set of allowable parameter trajectories

$$
\begin{gathered}
F_{\mathcal{R}}^{v}=\left\{p: p(t) \in \mathcal{R} \subset \mathbb{R}^{s}, p\left(t_{k}+\tau\right)=p\left(t_{k}\right), \tau \in[0, h(p(k)),\right. \\
\left.\left|p_{i}\left(t_{k+1}\right)-p_{i}\left(t_{k}\right)\right| \leq v_{i}, i=1,2, \ldots, s\right\}
\end{gathered}
$$


where $t_{k}$ are sample instances, $h$ is the sampling interval and $\mathcal{R}$ is a compact subset of $\mathbb{R}^{s}$. So, $p(t)$ is assumed to be a bounded piecewiseconstant vector-valued function with bounded variation rates. It is assumed that at the sampling instances $t_{k}, k=0,1,2, \ldots$, the parameter vector $p(t)$ is measurable. We seek to design sampled-data control schemes that depend on the measurement of the parameter vector $p(t)$ to guarantee stability and performance of the closed-loop system, see Fig. 1. In this formulation, the controller $K(p)$ is a state-feedback control gain that is scheduled based on $p, S_{h(p)}$ is an ideal sampler (with sampling interval $h(p)$ ), and $H_{h(p)}$ is a zero-order hold (with sampling interval $h(p)$ ). The sampling interval $h$ is a bounded continuous function of the parameter vector $p$. The sampling and hold operators $S_{h(p)}$ and $H_{h(p)}$ are assumed to be synchronized. We call $H_{h(p)} K(p) S_{h(p)}$ the sampled-data LPV controller.

The lifting technique plays a key role in solving sampled-data control problems [3]. The sampled-data LPV control system in Fig. 1 can be converted to the following discrete-time LPV control system in Fig. 2,

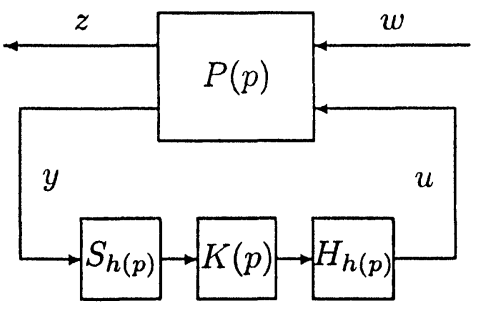

FIGURE 1 LPV sampled-data control system.

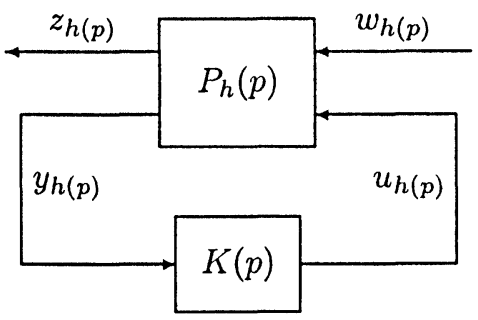

FIGURE 2 Lifted LPV sampled-data control system. 
where

$$
P_{h}(p)=\left[\begin{array}{cc}
W_{h(p)} & 0 \\
0 & S_{h(p)}
\end{array}\right] P(p)\left[\begin{array}{cc}
W_{h(p)}^{-1} & 0 \\
0 & H_{h(p)}
\end{array}\right],
$$

and $W_{h}$ is the lifting operator defined by $W_{h(p)}: L^{p}[0, \infty) \rightarrow l_{L^{p}[0, h(p)]}^{p}$ such that

$$
f_{h(p)}=W_{h(p)} f, \quad f_{h(p)}(k)(\tau)=f\left(t_{k}+\tau\right), \quad \tau \in\left[0, h\left(p\left(t_{k}\right)\right)\right) .
$$

Notice that although lifting techniques applied to sampled-data systems with constant sampled-period result in a discrete-time LTI representation, in our case where the sampling interval is a function of the parameter vector $p$ we obtain a discrete-time LPV representation.

The lifting operator $W_{h}$ provides an isometric mapping between the continuous and discrete normed spaces. In this paper, we will consider the signal spaces whose norms are the $L^{p}$ and $l^{p}$ norms for $p=2$ (energy of a signal) and $\infty$ (peak value of a signal). The lifted system $P_{h}(p)$ has a state-space realization,

$$
\begin{aligned}
x_{h}(k+1) & =A_{h}(p(k)) x_{h}(k)+B_{h 1}(p(k)) w_{h}(k)+B_{h 2}(p(k)) u_{h}(k) \\
z_{h}(k) & =C_{h 1}(p(k)) x_{h}(k)+D_{h 11}(p(k)) w_{h}(k)+D_{h 12}(p(k)) u_{h}(k) \\
y_{h}(k) & =x_{h}(k)
\end{aligned}
$$

where $\quad x_{h}(k)=x\left(t_{k}\right), \quad u_{h(k)}=u\left(t_{k}\right), \quad y_{h}(k)=y\left(t_{k}\right), \quad w_{h}=W_{h(p)} w, \quad z_{h}=$ $W_{h(p)} z$. The state-space matrices $A_{h}(\cdot), B_{h 2}(\cdot)$ are matrix valued functions, while $B_{h 1}(\cdot), C_{h 1}(\cdot), D_{h 11}(\cdot)$ and $D_{h 12}(\cdot)$ are operator valued. All these operators have finite rank. The system $P_{h}(p)$ is an infinite dimensional LPV system. In our approach, the control design problem for $P_{h}(p)$ will be transformed to a problem involving a finite-dimensional input/output plant that depends on the parameter vector $p$. Then, an LPV control design scheme will be implemented for control synthesis.

\section{LPV SAMPLED-DATA ENERGY-TO-ENERGY GAIN ANALYSIS}

Consider the unforced LPV system

$$
\dot{x}(t)=A(p(t)) x(t)
$$


where $p(\cdot) \in F_{\mathcal{R}}^{v}$ and $A(\cdot)$ is a bounded continuous function of $p$. Since $p(\cdot)$ is a piecewise-constant function of $t$, so will be $A(\cdot)$. The system (3) has a lifted representation

$$
x_{h}(k+1)=A_{h}(p(k)) x_{h}(k)
$$

where $x_{h}(k)$ is the $k$ th component of the lifted state vector and $A_{h}(p)=\exp (h(p) A(p))$.

The following result provides conditions for asymptotic stability of the LPV system (3) in terms of the lifted system (4).

THEOREM 1 Consider the unforced LPV system (3) and $A_{h}(p)$ defined in (4). If there exists a bounded continuous matrix function $Q(p)>0$, such that

$$
A_{h}^{\prime}(p) Q(p) A_{h}(p)-Q(p+q)<0
$$

for all $p \in \mathcal{R}, p+q \in \mathcal{R}$ and $\left|q_{i}\right| \leq v_{i}$, then the system (3) is asymptotically stable, that is, the solution $x(\cdot)$ converges to zero as $t \rightarrow \infty$ for all $p(\cdot) \in F_{\mathcal{R}}$.

Proof We first show that the system (4) is asymptotically stable if (5) holds for some matrix function $Q(p)>0$. Let $V(k)=x_{h}^{\mathrm{T}}(k) \times$ $Q(p(k-1)) x_{h}(k)$. Because of the compactness of $\mathcal{R}$, it is true that

$$
\begin{aligned}
& V(k) \geq \inf _{p \in \mathcal{R}} \lambda_{\min }(Q(p))\left\|x_{h}(k)\right\|_{2}^{2}=\min _{p \in \mathcal{R}} \lambda_{\min }(Q(p))\left\|x_{h}(k)\right\|_{2}^{2} \\
& V(k) \leq \sup _{p \in \mathcal{R}} \lambda_{\max }(Q(p))\left\|x_{h}(k)\right\|_{2}^{2}=\max _{p \in \mathcal{R}} \lambda_{\max }(Q(p))\left\|x_{h}(k)\right\|_{2}^{2} .
\end{aligned}
$$

Notice that $p(k-1)=p(k)+q(k)$ for some $q(k)$ with $\left|q_{i}(k)\right| \leq v_{i}$. Hence,

$$
\begin{aligned}
\Delta V(k)= & V(k+1)-V(k) \\
= & x_{h}^{\prime}(k+1) Q(p(k)) x_{h}^{\prime}(k+1)-x_{h}^{\prime}(k) Q(p(k-1)) x_{h}(k) \\
= & x_{h}^{\prime}(k) A_{h}^{\prime}(p(k)) Q(p(k)) A_{h}(p(k)) x_{h}(k) \\
& -x_{h}^{\prime}(k) Q(p(k)+q(k)) x_{h}(k) \\
= & x_{h}^{\prime}(k)\left[A_{h}^{\prime}(p(k)) Q(p(k)) A_{h}(p(k))-Q(p(k)+q(k))\right] x_{h}(k) \\
\leq & \lambda_{\max }\left\|x_{h}(k)\right\|_{2}^{2} .
\end{aligned}
$$


The value of $\sup _{p \in \mathcal{R}, p+q \in \mathcal{R},\left|q_{i}\right| \leq v_{i}}\left[A_{h}^{\prime}(p(k)) Q(p(k)) A_{h}(p(k))-\right.$ $Q(p(k)+q(k))]$ is attainable since $\mathcal{R}$ is a compact subset of $\mathbb{R}^{s}$ and we denote this value by $\lambda_{\max }$. Note that because of (5), $\lambda_{\max }<0$. Hence, $V(k)$ is a Lyapunov function for (4). From Lyapunov stability theory [15], we obtain that (4) is asymptotically stable, that is, $x_{h}(k)$ converges to 0 as $k \rightarrow \infty$.

Now consider the state $x(t)$ of (3) between sampling instances. We have that

$$
\left\|x\left(t_{k}+\tau\right)\right\|_{2}=\left\|\mathrm{e}^{A(p(k)) \tau} x\left(t_{k}\right)\right\|_{2} \leq\left\|\mathrm{e}^{A(p(k)) \tau}\right\| \cdot\left\|x_{h}(k)\right\|_{2} .
$$

Notice that $\sup _{\tau \in[0, h], p \in \mathcal{R}}\left\|\mathrm{e}^{A(p(k)) \tau}\right\|=\sup _{\tau \in[0, h], p \in \mathcal{R}} \sigma_{\max }\left(\mathrm{e}^{A(p(k)) \tau}\right)$ is reached since it is the supremum of a bounded continuous function on the compact set $[0, h] \times \mathcal{R}$. If we demote this supremum by $M$, then

$$
\left\|x\left(t_{k}+\tau\right)\right\|_{2} \leq M\left\|x_{h}(k)\right\|_{2} \quad \text { for } \tau \in[0, h(p(k))) .
$$

Hence, since $x_{h}(k)$ converges to 0 as $k \rightarrow \infty$, we obtain that $x(t)$ converges to 0 as $t \rightarrow \infty$. Therefore, the system (3) is asymtotically stable.

If the assumptions of Theorem 1 are satisfied, we will say that the LPV system is sampled-data parameter-dependent quadratically stable (or SDPDQ stable). Hence, SDPDQ stability implies asymptotic stability for the unforced LPV system in (3). Here we consider the matrix function $Q$ in an affine form

$$
Q(p)=Q_{0}+\sum_{i=1}^{s} p_{i} Q_{i}
$$

where $Q_{i}, i=0,1, \ldots, s$ are constant matrices. This matrix function is continuous and bounded for $p \in \mathcal{R}$.

COROllary 2 Consider the unforced LPV system (3) and $A_{h}(p)$ defined in (4). If there exists an affine function $Q(p)=Q_{0}+$ $\sum_{i=1}^{s} p_{i} Q_{i}>0$, such that

$$
A_{h}^{\prime}(p) Q(p) A_{h}(p)-Q(p)-\sum_{i=1}^{s} \pm\left(v_{i} Q_{i}\right)<0
$$

for all $p \in \mathcal{R}$, then the system (3) is SDPDQ stable. 
Remark 3 The notation $\sum_{i=1}^{s} \pm(\cdot)$ in (6) is used to indicate that every combination of $+(\cdot)$ and $-(\cdot)$ should be included in the inequality. That is, the inequality (6) represents $2^{s}$ different combinations in the summation.

To examine the energy-to-energy (induced $L^{2}$ norm) problem, now consider the following input-output LPV system

$$
\begin{aligned}
\dot{x}(t) & =A(p(t)) x(t)+B(p(t)) w(t) \\
z(t) & =C(p(t)) x(t)+D(p(t)) w(t)
\end{aligned}
$$

with $p \in F_{\mathcal{R}}^{v}$. This system can be lifted to the following discrete-time LPV representation

$$
\begin{aligned}
x_{h}(k+1) & =A_{h}(p(k)) x_{h}(k)+B_{h}(p(k)) w_{h}(k) \\
z_{h}(k) & =C_{h}(p(k)) x_{h}(k)+D_{h}(p(k)) w_{h}(k)
\end{aligned}
$$

where $w_{h}(k)$ and $z_{h}(k)$ are the $k$ th components of the lifted input/ output signals, and the operators $B_{h}$ and $C_{h}$ have finite rank. The following result provides conditions for the LPV system (7) to have energy-to-energy gain (induced $L^{2}$ norm) less that $\gamma$ in terms of the lifted system (8).

THEOREM 4 Consider the input-output LPV system in (7) with $p \in F_{\mathcal{R}}^{v}$ and its lifted representation (8) with $D_{h}^{*} D_{h}-\gamma^{2} I<0$. Then (7) is $S D P D Q$ stable and has energy-to-energy gain less than $\gamma$ if there exists a bounded continuous matrix function $Q(p)>0$, such that

$$
\left[\begin{array}{cc}
A_{d}^{\prime}(p) Q(p) A_{d}(p)-Q(p+q) & A_{d}^{\prime}(p) Q(p) B_{d}(p) \\
+C_{d}^{\prime}(p) C_{d}(p) & \\
B_{d}^{\prime}(p) Q(p) A_{d}(p) & B_{d}^{\prime}(p) Q(p) B_{d}(p)-\gamma^{2} I
\end{array}\right]<0
$$

where $A_{d}(p), B_{d}(p)$ and $C_{d}(p)$ are matrix valued function satisfying

$$
\begin{aligned}
A_{d}(p) & =A_{h}(p)+B_{h}(p) D_{h}^{*}(p)\left(\gamma^{2} I-D_{h}(p) D_{h}^{*}(p)\right)^{-1} C_{h}(p) \\
B_{d}(p) B_{d}^{\prime}(p) & =\gamma^{2} B_{h}(p)\left(\gamma^{2} I-D_{h}^{*}(p) D_{h}(p)\right)^{-1} B_{h}^{*}(p) \\
C_{d}^{\prime}(p) C_{d}(p) & =\gamma^{2} C_{h}^{*}(p)\left(\gamma^{2} I-D_{h}(p) D_{h}^{*}(p)\right)^{-1} C_{h}(p)
\end{aligned}
$$

for all $p \in \mathcal{R}, p+q \in \mathcal{R}$ and $\left|q_{i}\right| \leq v_{i}, i=1,2, \ldots, s$. 
Proof Condition (9) implies that the $(1,1)$ block in the left-hand side of (9) is negative definite. Hence, Theorem 1 guarantees that the system (7) is SDPDQ stable. We consider now the energy-to-energy gain of system (8). The Schur complement formula implies the following condition from (9)

$$
A_{d}^{\prime} Q A_{d}-Q(p+q)+A_{d}^{\prime} Q B_{d}\left(\gamma^{2} I-B_{d}^{\prime} Q B_{d}\right)^{-1} B_{d}^{\prime} Q A_{d}+C_{d}^{\prime} C_{d}<0 .
$$

The matrix inversion lemma

$$
(A+B C D)^{-1}=A^{-1}-A^{-1} B\left(C^{-1}+D A^{-1} B\right)^{-1} D A^{-1}
$$

and the expressions (10) provide the following result.

$$
\begin{aligned}
& A_{h}^{\prime} Q A_{h}+C_{h}^{*} C_{h}+\left(A_{h}^{\prime} Q B_{h}+C_{h}^{*} D_{h}\right) \\
& \quad \times\left(\gamma^{2} I-B_{h}^{*} Q B_{h}-D_{h}^{*} D_{h}\right)^{-1}\left(B_{h}^{*} Q A_{h}+D_{h}^{*} C_{h}\right) \\
& \quad=A_{d}^{\prime} Q A_{d}+C_{d}^{\prime} C_{d}+A_{d}^{\prime} Q B_{d}\left(\gamma^{2} I-B_{d}^{\prime} Q B_{d}\right)^{-1} B_{d}^{\prime} Q A_{d} .
\end{aligned}
$$

Hence, (11) results in

$$
\begin{aligned}
& A_{h}^{\prime} Q A_{h}-Q(p+q)+\left(A_{h}^{\prime} Q B_{h}+C_{h}^{*} D_{h}\right)\left(\gamma^{2} I-B_{h}^{*} Q B_{h}-D_{h}^{*} D_{h}\right)^{-1} \\
& \quad \times\left(B_{h}^{*} Q A_{h}+D_{h}^{*} C_{h}\right)+C_{h}^{*} C_{h}<0 .
\end{aligned}
$$

Now notice that (9) implies

$$
B_{d}^{\prime} Q B_{d}-\gamma^{2} I<0
$$

From the Schur complement formula, this is equivalent to

$$
\left[\begin{array}{cc}
Q^{-1} & B_{d} \\
B_{d}^{\prime} & \gamma^{2} I
\end{array}\right]>0 .
$$

Note the relation in (10) and using the Schur complement formula again, we get

$$
\left[\begin{array}{cc}
\gamma^{-2} Q^{-1} & B_{h} \\
B_{h}^{*} & I-\gamma^{-2} D_{h}^{*} D_{h}
\end{array}\right]>0
$$


so

$$
\gamma^{2} I-D_{h}^{*} D_{h}-B_{h}^{*} Q B_{h}>0 .
$$

Hence, applying again the Schur complement formula we obtain from (12) and (13)

$$
\left[\begin{array}{cc}
A_{h}^{\prime} Q A_{h}-Q(p+q)+C_{h}^{*} C_{h} & A_{h}^{\prime} Q B_{h}+C_{h}^{*} D_{h} \\
B_{h}^{*} Q A_{h}+D_{h}^{*} C_{h} & B_{h}^{*} Q B_{h}+D_{h}^{*} D_{h}-\gamma^{2} I
\end{array}\right]<0 .
$$

Multiplying by $\left[x_{h}^{\prime}(k) w_{h}^{*}(k)\right]$ and its adjoint from left and right, respectively, we obtain

$$
\begin{aligned}
& x_{h}^{\prime}(k+1) Q(p(k)) x_{h}(k+1)-x_{h}^{\prime}(k) Q(p(k-1)) x_{h}(k) \\
& \quad+z_{h}^{*}(k) z_{h}(k)-\gamma^{2} w_{h}^{*}(k) w_{h}(k)<0 .
\end{aligned}
$$

Now, take the summation from $k=0$ to $k=\infty$, and since system (7) is SDPDQ stable, that is $\lim _{k \rightarrow \infty} x_{h}(k)=0$, we obtain

$$
\left\|z_{h}\right\|_{2}^{2}<\gamma^{2}\left\|w_{h}\right\|_{2}^{2}
$$

i.e., the energy-to-energy gain of the system is less than $\gamma$.

If the assumptions of Theorem 4 are satisfied, we will say that the LPV sampled-data system is SDPDQ stable and has energy-to-energy gain less than $\gamma$.

Remark 5 The condition $D_{h}^{*} D_{h}-\gamma^{2} I<0$ can be checked by matrix computation [5], and if it is true, the expressions in (10) are well defined. $C_{h}(\cdot), B_{h}(\cdot), D_{h}(\cdot)$ are operators but their compositions in the right-hand side of $(10)$ are matrix valued for fixed $p$. Then, $B_{d}(\cdot)$ and $C_{d}(\cdot)$ can be obtained by a matrix factorization, for example, the Cholesky factorization. By gridding the parameter space $\mathcal{R}$, the condition (9) can be tested via a finite dimensional LMI optimization.

COROllary 6 Consider the input-output LPV system in (7) with $p \in F_{\mathcal{R}}^{v}$ and its lifted representation (8) with $D_{h}^{*} D_{h}-\gamma^{2} I<0$. Then (7) is 
$S D P D Q$ stable and has energy-to-energy gain less than $\gamma$ if there exists an affine matrix function $Q(p)=Q_{0}+\sum_{i=1}^{s} p_{i} Q_{i}>0$, such that for all $p \in \mathcal{R}$

$$
\left[\begin{array}{cc}
A_{d}^{\prime}(p) Q(p) A_{d}(p)-Q(p) & A_{d}^{\prime}(p) Q(p) B_{d}(p) \\
-\sum_{i=1}^{s} \pm\left(v_{i} Q_{i}\right)+C_{d}^{\prime}(p) C_{d}(p) & \\
B_{d}^{\prime}(p) Q(p) A_{d}(p) & B_{d}^{\prime}(p) Q(p) B_{d}(p)-\gamma^{2} I
\end{array}\right]<0 .
$$

\section{LPV SAMPLED-DATA ENERGY-TO-ENERGY GAIN CONTROL DESIGN}

Consider the system (2), which has the same energy-to-energy gain as the system (1). We assume that $y_{h}(k)=x_{h}(k)$, that is, the states are available at the sampling times for feedback. The LPV sampled-data energy-to-energy control synthesis problem is to design a parameter varying controller $K(p)$ with a lifted representation

$$
u_{h}(k)=F(p(k)) x_{h}(k)
$$

to render the closed-loop system SDPDQ stable and to guarantee an energy-to-energy gain less than a given bound $\gamma$. The sampled-data state-space synthesis problem can be converted to a discrete-time state-feedback LPV synthesis problem using the following result.

LEMMA 7 Suppose $D_{h 11}^{*} D_{h 11}-\gamma^{2} I<0$. The state-feedback gain $F(p)$ in (15) makes the sampled-data system (2) SDPDQ stable with energyto-energy gain less than $\gamma$ if and only if the same state-feedback gain $F(p)$ makes the following discrete-time system (16) PDQ stable with energy-to-energy gain less than $\gamma$

$$
\begin{aligned}
x_{d}(k+1) & =A_{d}(p(k), \gamma) x_{d}(k)+B_{d 1}(p(k), \gamma) w_{d}(k)+B_{d 2}(p(k), \gamma) u_{d}(k) \\
z_{d}(k) & =C_{d 1}(p(k), \gamma) x_{d}(k)+D_{d 12}(p(k), \gamma) u_{d}(k)
\end{aligned}
$$


with state-space matrices satisfying

$$
\begin{aligned}
& A_{d}(p)= A_{h}(p)+B_{h 1}(p) D_{h 1}^{*}(p)\left(\gamma^{2} I-D_{h 11}(p) D_{h 11}^{*}(p)\right)^{-1} C_{h}(p) \\
& B_{d 2}(p)= B_{h 2}(p)+B_{h 1}(p) D_{h 1}^{*}(p)\left(\gamma^{2} I-D_{h 11}(p)\right. \\
&\left.\times D_{h 11}^{*}(p)\right)^{-1} D_{h 12}(p) \\
& B_{d 1}(p) B_{d 1}^{\prime}(p)= \gamma^{2} B_{h 1}(p)\left(\gamma^{2} I-D_{h 11}^{*}(p) D_{h 11}(p)\right)^{-1} B_{h 1}^{*}(p) \\
& {\left[\begin{array}{c}
C_{d 1}^{\prime}(p) \\
D_{d 12}^{\prime}(p)
\end{array}\right]\left[\begin{array}{c}
C_{d 1}^{\prime}(p) \\
D_{d 12}^{\prime}(p)
\end{array}\right]^{\prime} } \\
&=\gamma^{2}\left[\begin{array}{c}
C_{h 1}^{*}(p) \\
D_{h 12}^{*}(p)
\end{array}\right]\left(\gamma^{2} I-D_{h 11}(p) D_{h 11}^{*}(p)\right)^{-1}\left[\begin{array}{c}
C_{h 1}^{*}(p) \\
D_{h 12}^{*}(p)
\end{array}\right]^{*}
\end{aligned}
$$

and the state-feedback controller is

$$
u_{d}(k)=F(p(k)) x_{d}(k)
$$

Proof The closed loop system for the plant (2) and the control (15) is

$$
\begin{aligned}
x_{h}(k+1) & =\left(A_{h}(p(k))+B_{h 2}(p(k)) F(p(k))\right) x_{h}(k)+B_{h 1}(p(k)) w_{h}(k) \\
z_{h}(k) & =\left(C_{h 1}(p(k))+D_{h 12}(p(k)) F(p(k))\right) x_{h}(k)+D_{h 11}(p(k)) w_{h}(k) .
\end{aligned}
$$

On the other hand, the closed loop system for the plant (16) and the control (18) is

$$
\begin{aligned}
x_{d}(k+1) & =\left(A_{d}(p(k))+B_{d 2}(p(k)) F(p(k))\right) x_{d}(k)+B_{d 1}(p(k)) w_{d}(k) \\
z_{d}(k) & =\left(C_{d 1}(p(k))+D_{d 12}(p(k)) F(p(k))\right) x_{d}(k)
\end{aligned}
$$

By Theorem 4 the energy-to-energy gain of the two systems is less than $\gamma$ noting that (17) implies that (10) holds for the two closed loop systems.

For simplicity, we assume that $D_{d 12}(p)$ has full column rank for all $p \in \mathcal{R}$. Then the system can be expressed in a form where

$$
D_{d 12}=\left[\begin{array}{l}
0 \\
I
\end{array}\right], \quad C_{d 1}=\left[\begin{array}{l}
C_{d 11} \\
C_{d 12}
\end{array}\right] \text {. }
$$


The following result provides the synthesis conditions for the energyto-energy gain sample-data LPV control problem.

THEOREM 8 Suppose $D_{h 11}^{*} D_{h 11}-\gamma^{2} I<0$. The state-feedback gain $F(p)$ in (15) makes the system (2) SDPDQ stable with energy-to-energy gain less than $\gamma$ if and only if there exists a matrix function $S(p)>0$, such that for all $p \in \mathcal{R}, p+q \in \mathcal{R},\left|q_{i}\right| \leq v_{i}, i=1,2, \ldots, s$

$\left[\begin{array}{ccc}-S(p+q) & S(p+q)\left(A_{d}^{\prime}(p)\right. & S(p+q) C_{d 11}^{\prime}(p) \\ & \left.-C_{d 12}^{\prime}(p) B_{d 1}^{\prime}(p)\right) & \\ \left(A_{d}(p)-B_{d 1}(p) C_{d 12}(p)\right) S(p+q) & -S(p)+\gamma^{-2} B_{d 1}(p) B_{d 1}^{\prime}(p) & 0 \\ C_{d 11}(p) S(p+q) & -B_{d 2}(p) B_{d 2}^{\prime}(p) & \\ & 0 & -I\end{array}\right]<0$

$$
-S(p)+\gamma^{-2} B_{d 1}(p) B_{d 1}^{\prime}(p)<0 .
$$

In this case, the state-feedback gain is

$$
\begin{aligned}
F(p)= & -\left[I+B_{d 2}^{\prime}(p)\left(S(p)-\gamma^{-2} B_{d 1}(p) B_{d 1}^{\prime}(p)\right)^{-1} B_{d 2}(p)\right]^{-1} \\
& \times\left[B_{d 2}^{\prime}(p)\left(S(p)-\gamma^{-2} B_{d 1}(p) B_{d 1}^{\prime}(p)\right)^{-1} A_{d}(p)+C_{d 12}(p)\right]
\end{aligned}
$$

Proof First we proof the necessity part. From Lemma 7, we only need to investigate the discrete-time LPV system (16) instead of the sampled-data system (2). The closed loop system of (16) and (18) is

$$
\begin{aligned}
x_{d}(k+1) & =A_{f} x_{d}(k)+B_{d 1} w_{d}(k) \\
z_{d}(k) & =C_{f} x_{d}(k)
\end{aligned}
$$

where

$$
\begin{aligned}
& A_{f}=A_{d}+B_{d 2} F \\
& C_{f}=C_{d 1}+D_{d 12} F .
\end{aligned}
$$


From Theorem 4, we know that the system (22) is SDPDQ stable and has energy-to-energy gain less than $\gamma$ if and only if there exists a bounded continuous matrix function $Q(p)>0$, such that

$$
\left[\begin{array}{cc}
A_{f}^{\prime} Q A_{f}-Q(p+q)+C_{f}^{\prime} C_{f} & A_{f}^{\prime} Q B_{d 1} \\
B_{d 1}^{\prime} Q A_{f} & B_{d 1}^{\prime} Q B_{d 1}-\gamma^{2} I
\end{array}\right]<0 .
$$

Let $S(p)=Q^{-1}(p)$, and $S(p)>0$ is bounded continuous as $Q(p)$ is. Then (23) can be rewritten as

$$
\left[\begin{array}{cc}
-S^{-1}(p+q)+C_{f}^{\prime} C_{f} & 0 \\
0 & -\gamma^{2} I
\end{array}\right]+\left[\begin{array}{c}
A_{f}^{\prime} \\
B_{d 1}^{\prime}
\end{array}\right] S^{-1}\left[\begin{array}{ll}
A_{f} & B_{d 1}
\end{array}\right]<0 .
$$

Applying the Schur complement formula, this is equivalent to

$$
\left[\begin{array}{cccc}
-S^{-1}(p+q) & 0 & A_{f}^{\prime} & C_{f}^{\prime} \\
0 & -\gamma^{2} I & B_{d 1}^{\prime} & 0 \\
A_{f} & B_{d 1} & -S & 0 \\
C_{f} & 0 & 0 & -I
\end{array}\right]<0
$$

Pre- and post-multiplying this inequality by $S(p+q)$ in first row and column, we obtain

$$
\left[\begin{array}{cccc}
-S(p+q) & 0 & S(p+q) A_{d}^{\prime} & S(p+q) C_{d 1}^{\prime} \\
& & +S(p+q) F^{\prime} B_{d 2}^{\prime} & +S(p+q) F^{\prime} D_{d 12}^{\prime} \\
0 & -\gamma^{2} I & B_{d 1}^{\prime} & 0 \\
A_{d} S(p+q) & B_{d 1} & -S & 0 \\
+B_{d 2} F S(p+q) & & 0 & -I \\
C_{d 1} S(p+q) & 0 & & \\
+D_{d 12} F S(p+q) & & &
\end{array}\right]<0
$$


From the Schur complement formula, this is equivalent to

$$
\left[\begin{array}{ccc}
-S(p+q) & S(p+q) A_{d}^{\prime} & S(p+q) C_{d 1}^{\prime} \\
& +S(p+q) F^{\prime} B_{d 2}^{\prime} & +S(p+q) F^{\prime} D_{d 12}^{\prime} \\
A_{d} S(p+q) & -S+\gamma^{-2} B_{d 1} B_{d 1}^{\prime} & 0 \\
+B_{d 2} F S(p+q) & & \\
C_{d 1} S(p+q) & 0 & -I \\
+D_{d 12} F S(p+q) & &
\end{array}\right]<0 .
$$

We write it in a basic LMI form

$$
V F W+W^{\prime} F^{\prime} V^{\prime}+\theta<0
$$

where

$$
\begin{aligned}
& \theta=\left[\begin{array}{ccc}
-S(p+q) & S(p+q) A_{d}^{\prime} & S(p+q) C_{d 1}^{\prime} \\
A_{d} S(p+q) & -S+\gamma^{-2} B_{d 1} B_{d 1}^{\prime} & 0 \\
C_{d 1} S(p+q) & 0 & -I
\end{array}\right] \\
& V=\left[\begin{array}{lll}
0 & B_{d 2}^{\prime} & D_{d 12}^{\prime}
\end{array}\right]^{\prime}, \quad W=\left[\begin{array}{lll}
S(p+q) & 0 & 0
\end{array}\right] .
\end{aligned}
$$

Notice that

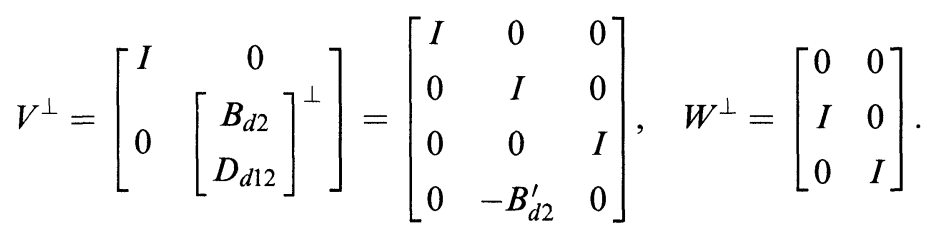

The solvability conditions for the basic LMI (25) are [13]

$$
\begin{aligned}
& V^{\perp^{\prime}} \theta V^{\perp}<0 \\
& W^{\perp^{\prime}} \theta W<0
\end{aligned}
$$

which are exactly (19) and (20).

Now we prove the sufficiency part. We seek to prove that the statefeedback gain (21) guarantees that (23) holds for $Q=S^{-1}$ under conditions (19) and (20). 
It is true that (20) is equivalent to

$$
B_{d 1}^{\prime} S^{-1} B_{d 1}-\gamma^{2} I<0 .
$$

Using the Schur complementary formula, (35) holds if we can prove that $M_{1}<0$ where

$$
\begin{aligned}
M_{1}= & -S(p+q)^{-1}+C_{d 11}^{\prime} C_{d 11}+\left(C_{d 12}^{\prime}+F^{\prime}\right)\left(C_{d 12}+F\right) \\
& -\left(A_{d}^{\prime}+F^{\prime} B_{d 2}^{\prime}\right)\left(-S+\gamma^{-2} B_{d 1} B_{d 1}^{\prime}\right)^{-1}\left(A_{d}+B_{d 2} F\right) .
\end{aligned}
$$

By solving

$$
\frac{\mathrm{d} M_{1}}{\mathrm{~d} F^{\prime}}=2 C_{d 12}+2 F+2 B_{d 2}^{\prime}\left(S-\gamma^{-2} B_{d 1} B_{d 1}^{\prime}\right)^{-1}\left(A_{d}+B_{d 2} F\right)=0
$$

we get (21). To prove that $M_{1}<0$ for such $F$, using the Schur complimentary formula for (19), we obtain

$$
\begin{aligned}
M_{2}= & -S(p+q)^{-1}+C_{d 11}^{\prime} C_{d 11}-\left(A_{d}^{\prime}-C_{d 12}^{\prime} B_{d 2}^{\prime}\right) \\
& \times\left(-S+\gamma^{-2} B_{d 1} B_{d 1}^{\prime}-B_{d 2} B_{d 2}^{\prime}\right)^{-1}\left(A_{d}-B_{d 2} C_{d 12}\right)<0
\end{aligned}
$$

It can be checked that $M_{1}=M_{2}$ for $F$ given by (21). Let

$$
\begin{aligned}
M= & M_{1}-M_{2} \\
= & \left(A_{d}^{\prime}-C_{d 12}^{\prime} B_{d 2}^{\prime}\right)\left(S-\gamma^{-2} B_{d 1} B_{d 1}^{\prime}+B_{d 2} B_{d 2}^{\prime}\right)^{-1}\left(A_{d}-B_{d 2} C_{d 12}\right) \\
& +C_{d 12}^{\prime} C_{d 12}+A_{d}^{\prime}\left(S-\gamma^{-2} B_{d 1} B_{d 1}^{\prime}\right)^{-1} A_{d} \\
& -\left(A_{d}^{\prime}\left(S-\gamma^{-2} B_{d 1} B_{d 1}^{\prime}\right)^{-1} B_{d 2}+C_{d 12}^{\prime}\right) \\
& \times\left(I+B_{d 2}^{\prime}\left(S-\gamma^{-2} B_{d 1} B_{d 1}^{\prime}\right)^{-1} B_{d 2}\right)^{-1} \\
& \times\left(B_{d 2}^{\prime}\left(S-\gamma^{-2} B_{d 1} B_{d 1}^{\prime}\right)^{-1} A_{d}+C_{d 12}^{\prime}\right) .
\end{aligned}
$$

$M=0$ can be verified by checking that the coefficients for the terms $C_{d 12}^{\prime} C_{d 12}, A_{d}^{\prime} A_{d}, C_{d 12}^{\prime} A_{d}$ in $M$ all equal 0 . For example

$$
\begin{aligned}
\left.M\right|_{C_{d 12}^{\prime} C_{d 12}=} & C_{d 12}^{\prime}\left[I-\left(S-\gamma^{-2} B_{d 1} B_{d 1}^{\prime}+B_{d 2} B_{d 2}^{\prime}\right)^{-1}\right. \\
& \left.-\left(I+B_{d 2}^{\prime}\left(S-\gamma^{-2} B_{d 1} B_{d 1}^{\prime}\right)^{-1} B_{d 2}\right)^{-1}\right] C_{d 12}^{\prime} \\
= & 0
\end{aligned}
$$


since

$$
\left(I+B_{d 2}^{\prime}\left(S-\gamma^{-2} B_{d 1} B_{d 1}^{\prime}\right)^{-1} B_{d 2}\right)^{-1}=I-\left(S-\gamma^{-2} B_{d 1} B_{d 1}^{\prime}+B_{d 2} B_{d 2}^{\prime}\right)^{-1} .
$$

Remark 9 The state-feedback gain (21) is same as the static state feedback law in [14] (where a minus sign is missing) by letting $S=P^{-1}$, where $P$ is the solution of an algebraic Riccati equation in [14].

Corollary 10 Suppose $D_{h 11}^{*} D_{h 11}-\gamma^{2} I<0$. The state-feedback gain $F(p)$ in (15) makes the system (2) SDPDQ stable with energy-to-energy gain less than $\gamma$ if there exists an affine matrix function $S(p)=S_{0}+$ $\sum_{i=1}^{s} p_{i} S_{i}>0$, such that for all $p \in \mathcal{R}$

$$
\left[\begin{array}{ccc}
-S(p)-\sum_{i=1}^{s} \pm\left(v_{i} S_{i}\right) & \left(S(p)+\sum_{i=1}^{s} \pm\left(v_{i} S_{i}\right)\right)\left(A_{d}^{\prime}(p)\right. & \left(S(p)+\sum_{i=1}^{s} \pm\left(v_{i} S_{i}\right)\right) \\
& \left.-C_{d 12}^{\prime}(p) B_{d 1}^{\prime}(p)\right) & \times C_{d 11}^{\prime}(p) \\
\left(A_{d}(p)-B_{d 1}(p) C_{d 12}(p)\right) & -S(p)+\gamma^{-2} B_{d 1}(p) B_{d 1}^{\prime}(p) & 0 \\
\times\left(S(p)+\sum_{i=1}^{s} \pm\left(v_{i} S_{i}\right)\right) & -B_{d 2}(p) B_{d 2}^{\prime}(p) & \\
C_{d 11}(p)\left(S(p)+\sum_{i=1}^{s} \pm\left(v_{i} S_{i}\right)\right) & 0 & -I
\end{array}\right]<0
$$

In this case, the state-feedback gain is given by (21).

\section{LPV SAMPLED-DATA ENERGY-TO-PEAK GAIN PROBLEM}

Now we consider the energy-to-peak gain problem for the following LPV system

$$
\begin{aligned}
\dot{x}(t) & =A(p(t)) x(t)+B(p(t)) w(t) \\
z(t) & =C(p(t)) x(t)
\end{aligned}
$$

with $p \in F_{\mathcal{R}}^{v}$. Note that the system (26) has no feedthrough term, otherwise this system will not have a finite energy-to-peak gain. 
Since the lifting operator also preserves the peak norm, the system (26) can be lifted to

$$
\begin{aligned}
x_{h}(k+1) & =A_{h}(p(k)) x_{h}(k)+B_{h}(p(k)) w_{h}(k) \\
z_{h}(k) & =C_{h}(p(k)) x_{h}(k)+D_{h}(p(k)) w_{h}(k) .
\end{aligned}
$$

Note that $D_{h}$ is nonzero even though there is no feedthrough term in the system (26).

THEOREM 11 Consider the input-output LPV system (26) with $p \in F_{\mathcal{R}}^{v}$ and its lifted form (27). The system (26) is SDPDQ stable and has an energy-to-peak gain less than or equal to $\gamma$ if there exists a bounded continuous matrix function $Q(p)>0$ such that

$$
\begin{gathered}
A_{d}(p) Q(p) A_{d}^{\prime}(p)-Q(p+q)+B_{d}(p) B_{d}^{\prime}(p)<0 \\
C_{d \tau}(p) Q(p) C_{d \tau}^{\prime}(p)-\gamma^{2} I<0
\end{gathered}
$$

for $\tau \in[0, h(p)), p \in \mathcal{R}, p+q \in \mathcal{R},\left|q_{i}\right| \leq v_{i}, i=1,2, \ldots, s$, where $A_{d}(p)$, $B_{d}(p), C_{d \tau}(p, \gamma)$ are matrix valued function satisfying

$$
\begin{aligned}
A_{d}(p) & =A_{h}(p) \\
B_{d}(p) B_{d}^{\prime}(p) & =B_{h}(p) B_{h}^{*}(p) \\
C_{d \tau}^{\prime}(p) C_{d \tau}(p) & =\gamma^{-2} C_{\tau}^{*}(p)\left(\gamma^{2} I-D_{\tau}(p) D_{\tau}^{*}(p)\right)^{-1} C_{\tau}(p)
\end{aligned}
$$

and $C_{\tau}(\cdot), D_{\tau}(\cdot)$ have the same definitions as $C_{h}(\cdot), D_{h}(\cdot)$ by substituting $h$ with $\tau$.

Proof The assumption $Q(p)>0$ and (28) guarantee that the system (26) is SDPDQ stable from the Theorem 1. We now consider the system (27) for its energy-to-peak gain since it is the same as the system (26). From the Schur complement formula and (30), condition (28) is equivalent to

$$
\left[\begin{array}{cc}
Q^{-1} & 0 \\
0 & I
\end{array}\right]-\left[\begin{array}{c}
A_{h}^{\prime} \\
B_{h}^{*}
\end{array}\right] Q^{-1}(p+q)\left[\begin{array}{ll}
A_{h} & B_{h}
\end{array}\right]>0
$$


Let $w_{h}$ be any signal having finite energy, and $x_{h}$ be the solution of (27). Then, multiply $\left[x_{h}^{\prime}(k) w_{h}^{*}(k)\right]$ and its adjoint from left and right respectively to (31) to obtain

$$
\begin{aligned}
& x_{h}^{\prime}(k) Q^{-1}(p(k)) x_{h}(k)+w_{h}^{*}(k) w_{h}(k) \\
& \quad-x_{h}^{\prime}(k+1) Q^{-1}(p(k+1)) x_{h}(k+1)<0 .
\end{aligned}
$$

Taking summation from $k=0$ to $k=n-1$, we have

$$
x_{h}^{\prime}(n) Q^{-1}(n) x_{h}(n)<\sum_{k=0}^{n-1}\left\|w_{h}(k)\right\|_{2}^{2} .
$$

Using the Schur compliment formula again, this is equivalent to

$$
\left[\begin{array}{cc}
\sum_{k=0}^{n-1}\left\|w_{h}(k)\right\|_{2}^{2} & x_{h}^{\prime}(n) \\
x_{h}(n) & Q(n)
\end{array}\right]>0
$$

which implies

$$
\begin{aligned}
& {\left[\begin{array}{cc}
1 & 0 \\
0 & C_{\tau}(n)
\end{array}\right]\left[\begin{array}{cc}
\sum_{k=0}^{n-1}\left\|w_{h}(k)\right\|_{2}^{2} & x_{h}^{\prime}(n) \\
x_{n}(n) & Q(n)
\end{array}\right]\left[\begin{array}{cc}
1 & 0 \\
0 & C_{\tau}^{*}(n)
\end{array}\right]} \\
& +\left[\begin{array}{c}
w_{\tau}^{*}(n) \\
D_{\tau}(n)
\end{array}\right]\left[\begin{array}{ll}
w_{\tau}(n) & \left.D_{\tau}^{*}(n)\right] \geq 0
\end{array}\right.
\end{aligned}
$$

or equivalently

$$
\left[\begin{array}{cc}
\sum_{k=0}^{n-1}\left\|w_{h}(k)\right\|_{2}^{2}+\left\|w_{\tau}(n)\right\|_{2}^{2} & z^{\prime}(h n+\tau) \\
z(h n+\tau) & C_{\tau}(n) Q(n) C_{\tau}^{*}(n)+D_{\tau}(n) D_{\tau}^{*}(n)
\end{array}\right] \geq 0 .
$$

Using the Schur compliment formula and noting that (29) implies

$$
\gamma^{2} I>C_{\tau}(n) Q(n) C_{\tau}^{*}(n)+D_{\tau}(n) D_{\tau}^{*}(n)
$$


we have that

$$
\|z(h n+\tau)\| \leq \gamma\|w[0, h n+\tau)\|_{2}
$$

holds for $\tau \in[0, h)$ and for all $n$, which means that the energy-to-peak gain is less than or equal to $\gamma$.

If the assumptions of Theorem 11 are satisfied, we will say that the LPV sampled-data system is SDPDQ stable and has energy-to-peak gain less than $\gamma$.

COROLlARY 12 Consider the input-output LPV system (26) with $p \in$ $F_{\mathcal{R}}^{v}$ and its lifted form (27). The system (26) is SDPDQ stable and has an energy-to-peak gain less than or equal to $\gamma$ if there exists an affine matrix function $Q(p)=Q_{0}+\sum_{i=1}^{s} p_{i} Q_{i}>0$ such that for all $\tau \in[0, h(p))$, $p \in \mathcal{R}$

$$
\begin{gathered}
A_{d}(p) Q(p) A_{d}^{\prime}(p)-Q(p)-\sum_{i=1}^{s} \pm\left(v_{i} Q_{i}\right)+B_{d}(p) B_{d}^{\prime}(p)<0 \\
C_{d \tau}^{\prime}(p) Q(p) C_{d \tau}^{\prime}(p)-\gamma^{2} I<0 .
\end{gathered}
$$

Next we consider the energy-to-peak gain sampled-data statefeedback control synthesis. We assume that $D_{11}$ in the system (1) is zero. The controller is assumed to have the state-feedback parametervarying form (15).

THEOREM 13 Suppose $D_{\tau 11}^{*} D_{\tau 11}-\gamma^{2} I<0$. The state-feedback gain $F(p)$ in (15) makes the system (1) SDPDQ stable with energy-to-energy gain less than $\gamma$ if and only if there exists a matrix function $\tilde{F}(p)$ and $a$ bounded continuous matrix function $Q(p)>0$ such that

$$
\begin{aligned}
& {\left[\begin{array}{cc}
-Q(p+q)+B_{d 1}(p) B_{d 1}^{\prime}(p) & A_{d}(p) Q(p)+B_{d 2}(p) \tilde{F}(p) \\
Q(p) A_{d}^{\prime}(p)+\tilde{F}^{\prime}(p) B_{d 2}^{\prime}(p) & -Q(p)
\end{array}\right]<0} \\
& {\left[\begin{array}{cc}
-\gamma^{2} I & C_{d \tau 1}(p) Q(p)+D_{d \tau 12}(p) \tilde{F}(p) \\
Q(p) C_{d \tau 1}^{\prime}(p)+\tilde{F}^{\prime}(p) D_{d \tau 12}^{\prime}(p) & -Q(p)
\end{array}\right]<0}
\end{aligned}
$$


for $\tau \in[0, h), p \in \mathcal{R}, p+q \in \mathcal{R},\left|q_{i}\right| \leq v_{i}, i=1,2, \ldots, s$, where $A_{d}(p)$, $B_{d 1}(p), B_{d 2}(p), C_{d \tau 1}(p), D_{d \tau 12}(p)$ are matrix valued function satisfying

$$
\begin{gathered}
A_{d}(p)=A_{h}(p) \\
B_{d 1}(p) B_{d 1}^{\prime}(p)=B_{h 1}(p) B_{h 1}^{*}(p) \\
B_{d 2}(p)=B_{h 2}(p) \\
{\left[\begin{array}{c}
C_{d \tau 1}^{\prime}(p, \gamma) \\
D_{d \tau 12}^{\prime}(p, \gamma)
\end{array}\right]\left[\begin{array}{c}
C_{d \tau 1}^{\prime}(p, \gamma) \\
D_{d \tau 12}^{\prime}(p, \gamma)
\end{array}\right]^{\prime}} \\
=\gamma^{2}\left[\begin{array}{c}
C_{\tau 1}^{*}(p) \\
D_{\tau 12}^{*}(p)
\end{array}\right]\left(\gamma^{2} I-D_{\tau 11}(p) D_{\tau 11}^{*}(p)\right)^{-1}\left[\begin{array}{c}
C_{\tau 1}^{*}(p) \\
D_{h \tau 12}^{*}(p)
\end{array}\right]^{*}
\end{gathered}
$$

and $C_{\tau 1}(\cdot), D_{\tau 11}(\cdot), D_{\tau 12}(\cdot)$ have the same definitions as $C_{h 1}(\cdot), D_{h 11}(\cdot)$, $D_{h 12}(\cdot)$ by substituting $h$ with $\tau$. If the conditions are satisfied, the statefeedback gain is $F(p)=\tilde{F}(p) Q(p)^{-1}$.

Proof The closed loop system for the plant (2) and the control (15) is

$$
\begin{aligned}
x_{h}(k+1) & =\left(A_{h}(p(k))+B_{h 2}(p(k)) F(p(k))\right) x_{h}(k)+B_{h 1}(p(k)) w_{h}(k) \\
z_{h}(k) & =\left(C_{h 1}(p(k))+D_{h 12}(p(k)) F(p(k))\right) x_{h}(k)+D_{h 11}(p(k)) w_{h}(k) .
\end{aligned}
$$

From Theorem 11, the closed loop system is SDPDQ stable and has energy-to-peak gain less or equal to $\gamma$ if and only if there exists a bounded continuous matrix function $Q(p)>0$ such that

$$
\begin{gathered}
\left(A_{d}+B_{d 2} F\right) Q\left(A_{d}+B_{d 2} F\right)^{\prime}-Q(p+q)+B_{d 1} B_{d 1}^{\prime}<0 \\
\left(C_{d \tau 1}+D_{d \tau 12} F\right) Q(p)\left(C_{d \tau 1}+D_{d \tau 12} F\right)^{\prime}-\gamma^{2} I<0
\end{gathered}
$$

where $A_{d}, B_{d 1}, B_{d 2}, C_{d \tau 1}, D_{d \tau 12}$ are defined in (34). Using the Schur compliment formula, (35) and (36) can be written as

$$
\begin{gathered}
{\left[\begin{array}{cc}
-Q(p+q)+B_{d 1} B_{d 1}^{\prime} & \left(A_{d}+B_{d 2} F\right) Q \\
Q(p)\left(A_{d}+B_{d 2} F\right)^{\prime} & -Q
\end{array}\right]<0} \\
{\left[\begin{array}{cc}
-\gamma^{2} I & \left(C_{d \tau 1}+D_{d \tau 12} F\right) Q \\
Q\left(C_{d \tau 1}+D_{d \tau 12} F\right)^{\prime} & -Q
\end{array}\right]<0 .}
\end{gathered}
$$

Then (32) and (33) are obvious by letting $\tilde{F}=F Q$. 
COROLlaRY 14 Suppose $D_{\tau 11}^{*} D_{\tau 11}-\gamma^{2} I<0$. The state-feedback gain $F(p)$ in (15) makes the system (1) SDPDQ stable with energy-to-energy gain less than $\gamma$ if there exists a matrix function $\tilde{F}(p)$ and an affine matrix function $Q(p)=Q_{0}+\sum_{i=1}^{s} p_{i} Q_{i}>0$ such that

$$
\begin{gathered}
{\left[\begin{array}{cc}
-Q(p)-\sum_{i=1}^{s} \pm\left(v_{i} Q_{i}\right) & A_{d}(p) Q(p)+B_{d 2}(p) \tilde{F}(p) \\
+B_{d 1}(p) B_{d 1}^{\prime}(p) & \\
Q(p) A_{d}^{\prime}(p)+\tilde{F}^{\prime}(p) B_{d 2}^{\prime}(p) & -Q(p)
\end{array}\right]<0} \\
{\left[\begin{array}{cc}
-\gamma^{2} I & C_{d \tau 1}(p) Q(p)+D_{d \tau 12}(p) \tilde{F}(p) \\
Q(p) C_{d \tau 1}^{\prime}(p)+\tilde{F}^{\prime}(p) D_{d \tau 12}^{\prime}(p) & -Q(p)
\end{array}\right]<0}
\end{gathered}
$$

for all $\tau \in[0, h), p \in \mathcal{R}$. If the conditions are satisfied, the state-feedback gain is $F(p)=\tilde{F}(p) Q(p)^{-1}$.

\section{NUMERICAL EXAMPLE}

Consider the problem of designing a sampled-data controller to control the following plant

$$
\begin{aligned}
\dot{x}(t)= & {\left[\begin{array}{cc}
2 \sin (0.2 t) & 1.1+\sin (0.2 t) \\
-2.2+\sin (0.2 t) & -3.3+\sin (0.2 t)
\end{array}\right] x(t)+\left[\begin{array}{l}
0.2 \\
0.2
\end{array}\right] w(t) } \\
& +\left[\begin{array}{c}
2 \sin (0.2 t) \\
0.1+\sin (0.2 t)
\end{array}\right] u(t) \\
z(t)= & {\left[\begin{array}{ll}
0 & 1 \\
0 & 0
\end{array}\right] x(t)+\left[\begin{array}{l}
0 \\
1
\end{array}\right] u(t) . }
\end{aligned}
$$

We assume that the sine term in the above model corresponds to a plant parameter whose functional representation is not known a priori, 
but it can be measured in real-time. Hence, we define $p(t)=\sin (0.2 t)$, and the original system is formulated as an LPV system as follows

$$
\begin{aligned}
\dot{x}(t)= & {\left[\begin{array}{cc}
2 p(t) & 1.1+p(t) \\
-2.2+p(t) & -3.3+p(t)
\end{array}\right] x(t)+\left[\begin{array}{l}
0.2 \\
0.2
\end{array}\right] w(t) } \\
& +\left[\begin{array}{c}
2 p(t) \\
0.1+p(t)
\end{array}\right] u(t) \\
z(t)= & {\left[\begin{array}{ll}
0 & 1 \\
0 & 0
\end{array}\right] x(t)+\left[\begin{array}{l}
0 \\
1
\end{array}\right] u(t) . }
\end{aligned}
$$

The parameter $p(t) \in[-1,1]$. The sampling period $h$ will be chosen to be a constant $h=0.5$ for the proposed sampled-data scheme. Since $|\dot{p}(t)| \leq 0.2, p(t)$ does not change significantly within one sampling period. We use Corollary 10 to design a sampled-data parametervarying controller such that the closed-loop system is SDPDQ stable and has an energy-to-energy gain less than a criteria $\gamma=0.25$. For simplicity, we grid the parameter space using a 10 point grid. Solving the LMIs in Corollary 10, we get

$$
S=\left[\begin{array}{rr}
2.0656 & -0.5938 \\
-0.5938 & 2.7369
\end{array}\right]
$$

Given a unit rectangular disturbance $w(t)=1(t \in[0,5])$, we simulate the closed-loop system behavior. The output $z(t)$ is shown in Fig. 3 .

For comparison, we also consider a conventional approach to design a continuous-time LPV controller for the continuous-time plant, then discretize the controller. For the same energy-to-energy criteria $\gamma=$ 0.25 , we design a continuous-time LPV controller. The response is shown in Fig. 4. Then, we discretize the controller for several sampling periods: $h=0.2,0.25,0.3$. The response for the cases where $h=0.2$ and 0.25 are shown in Figs. 5 and 6. Notice that, when $h \geq 0.3$, the response diverges. The above comparison shows the advantage of the proposed sample-data LPV control design scheme. 


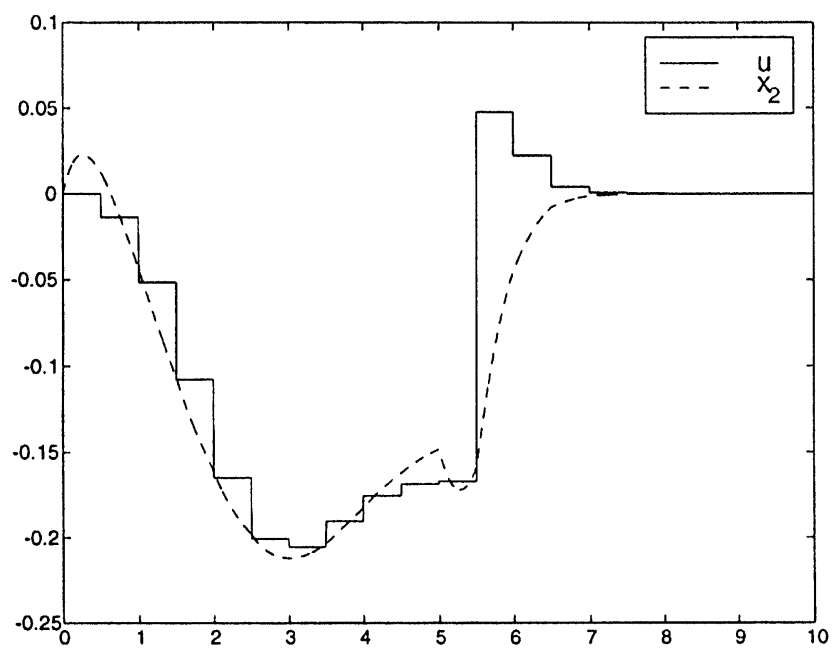

FIGURE 3 Sampled-data controller with $h=0.5$.

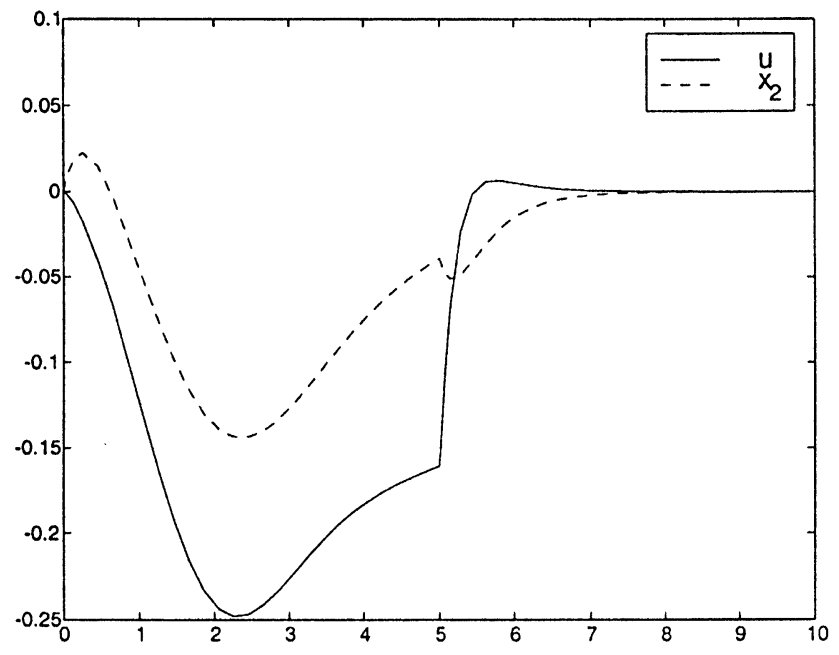

FIGURE 4 Continuous LPV controller.

\section{CONCLUSION}

In this work, the analysis and state-feedback synthesis problems for linear parameter-varying sampled-data systems have been examined. 


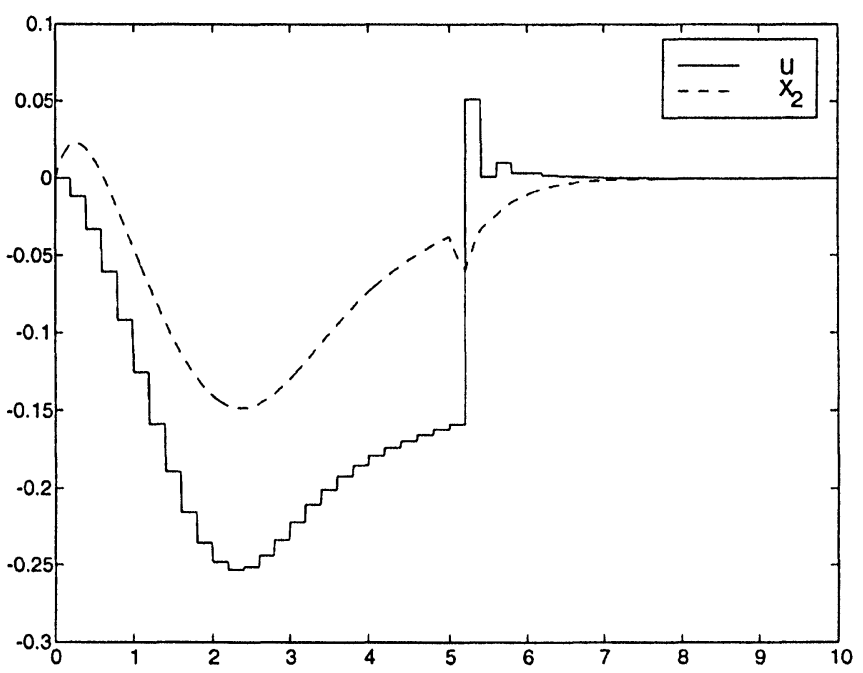

FIGURE 5 Discretized controller with $h=0.2$.

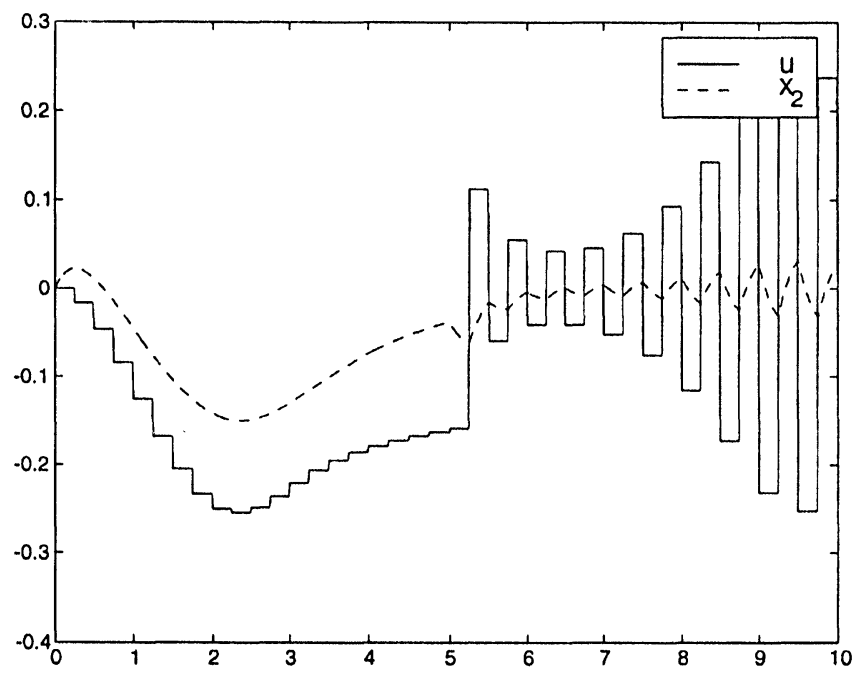

FIGURE 6 Discretized controller with $h=0.25$.

It is assumed that the system matrices and the sampling interval depend on system parameters that are measurable in real-time. The stabilization, energy-to-energy gain and energy-to-peak gain problems are considered. Using a lifting approach the analysis and synthesis problems 
are formulated as standard analysis and synthesis problems for an LPV discrete-time lifted system. The use of parameter-dependent Lyapunov functions provides LMI-based analysis and synthesis conditions that can be solved using efficient interior-point algorithms. The resulting discrete-time state-feedback controllers are scheduled based on the real-time measurement of the parameters. A numerical example demonstrates the advantage of the proposed approach compared to the traditional continuous-time design along with discretization.

\section{References}

[1] P. Apkarian and P. Gahinet. A convex characterization of gain-scheduled $H^{\infty}$ controllers, IEEE. Trans. Automatic Control, 40, 853-864, 1995.

[2] P. Apkarian and R.J. Adams. Advanced gain-scheduling techniques for uncertain systems, IEEE. Trans. Control Systems Technology, 6, 21-32, 1998.

[3] B.A. Bamieh and J.B. Pearson. A general framework for linear periodic systems with applications to $H^{\infty}$ sampled-data control, IEEE. Trans. Automatic Control, 37, 418-435, 1992.

[4] G. Becker and A.K. Packard. Robust performance of linear parametrically varying systems using parametrically-dependent linear feedback, Syst. Contr. Lett., 23, 205-215, 1994.

[5] T. Chen and B.A. Francis. Optimal Sampled-Data Control Systems, Springer, London, 1995.

[6] T. Chen and L. Qiu. $H^{\infty}$ design of general multirate sampled-data control systems, Automatica, 30, 1139-1152, 1994.

[7] S. Boyd, L. El Ghaoui, E. Feron and V. Balakrishnan. Linear Matrix Inequalities in Systems and Control Theory, SIAM Studies in Appl. Mathematics, Philadelphia, 1994.

[8] G.E. Dullerud. Control of Sampled-Data Systems, Birkhäuser, Boston, 1996.

[9] R.A. Horn and C.R. Johnson. Matrix Analysis, Cambridge University Press, 1985.

[10] A.K. Packard. Gain scheduling via linear fractional transformations, Syst. Contr. Lett., 22, 79-92, 1994.

[11] C.W. Scherer. Mixed $H^{2} / H^{\infty}$ control for time-varying and linear parametrically varying systems, Int. J. Robust Nonlin. Control., 6, 929-952, 1996.

[12] J.S. Shamma and M. Athans. Analysis of nonlinear gain-scheduled control systems, IEEE Trans. Automat. Contr., 35, 898-907, 1990.

[13] R.E. Skelton, T. Iwasaki and K.M. Grigoriadis. A Unified Algebraic Approach to Linear Control Design. Taylor \& Francis, London, 1998.

[14] A.A. Stoorvogel. The $H^{\infty}$ Control Problem: A State Space approach. Prentice-Hall, 1992.

[15] M. Vidyasagar. Nonlinear Systems Analysis, Prentice-Hall, 1993.

[16] F. Wu, X.H. Yang, A.K. Packard and G. Becker. Induced $L^{2}$ norm control for LPV systems with bounded parameter variation rates, Int. J. Robust Nonlin. Control., 6, 983-998, 1996. 


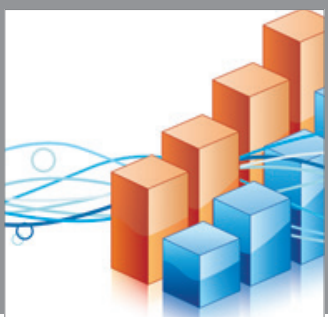

Advances in

Operations Research

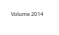

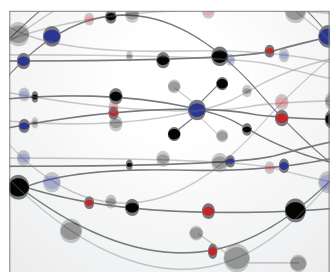

\section{The Scientific} World Journal
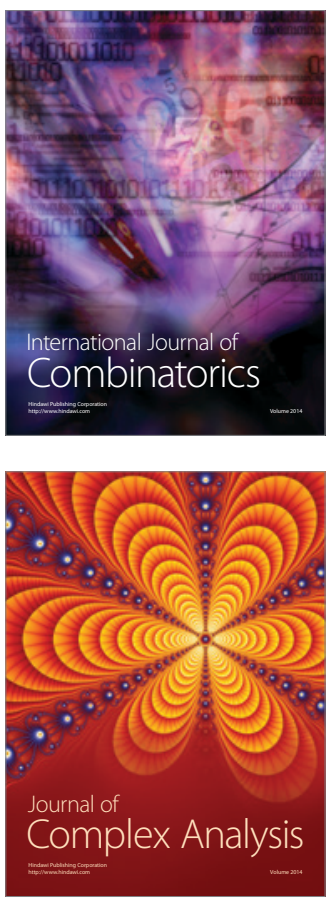

International Journal of

Mathematics and

Mathematical

Sciences
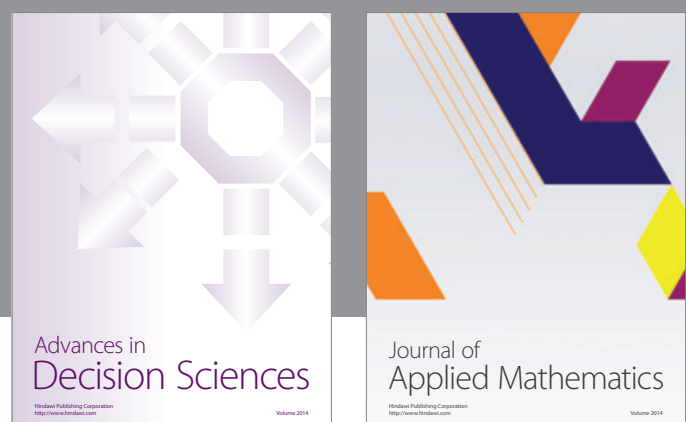

Journal of

Applied Mathematics
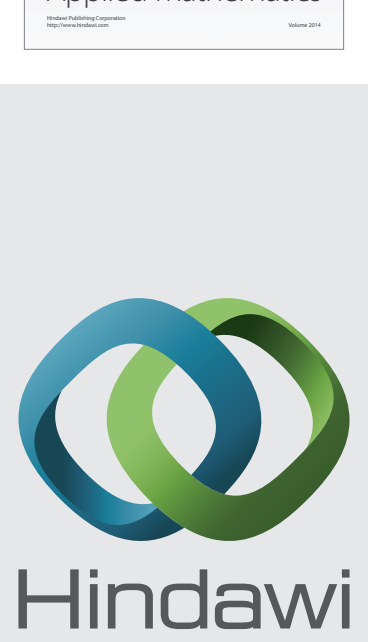

Submit your manuscripts at http://www.hindawi.com
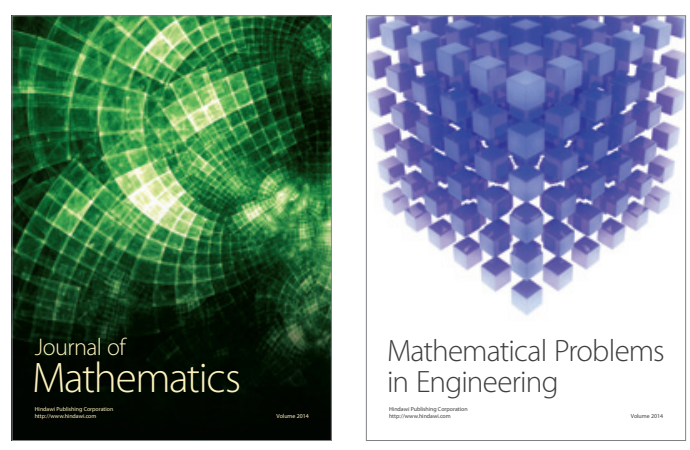

Mathematical Problems in Engineering
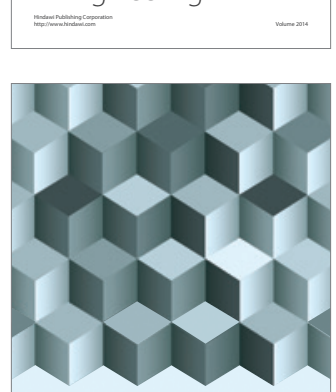

Journal of

Function Spaces
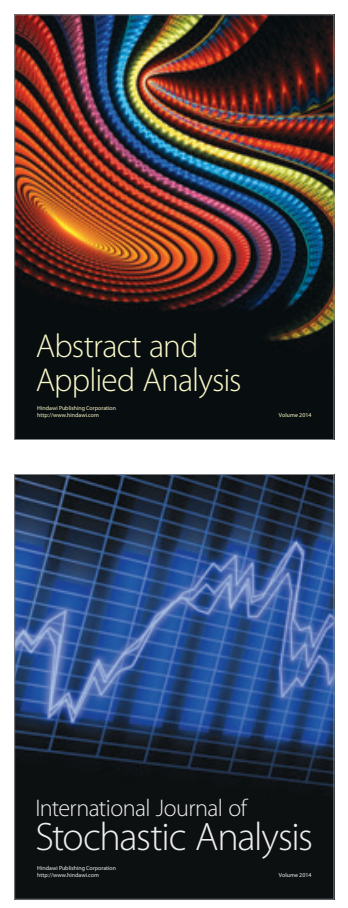

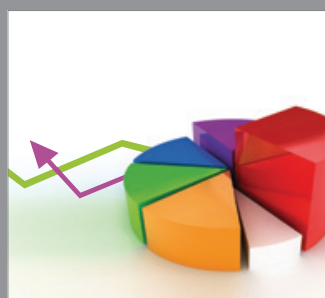

ournal of

Probability and Statistics

Promensencen
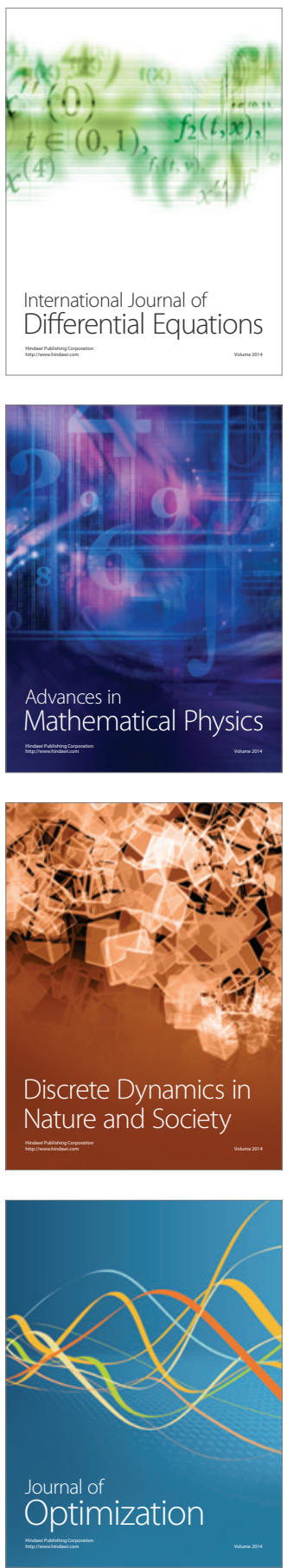